\title{
Cetuximab-induced aseptic meningitis: case report and review of a rare adverse event
}

\author{
Christophe Maritaz $^{1 *}$, Carole Metz ${ }^{1}$, Nabil Baba-Hamed ${ }^{2}$, Méryam Jardin-Szucs ${ }^{1}$ and Gaël Deplanque ${ }^{3}$
}

\begin{abstract}
Background: Cetuximab is a commonly used antibody agent in the treatment of colorectal or head and neck cancer. Although it is generally well tolerated in most patients, cetuximab has been associated with some rare but serious adverse events. Aseptic meningitis is one such distinctly uncommon adverse drug reaction.

Case presentation: We present the case of a middle-aged Caucasian patient, who presented with fever and headache within a few hours of starting cetuximab therapy and was diagnosed with cetuximab-induced aseptic meningitis after a complete workup.

Conclusion: To our knowledge, this is the ninth case of cetuximab-induced aseptic meningitis reported in literature. Because of a nonspecific clinical presentation, this adverse drug reaction can be easily misdiagnosed. It is important to increase awareness of this potentially severe reaction among oncologists.
\end{abstract}

Keywords: Cetuximab, Aseptic meningitis, Cancer

\section{Background}

Cetuximab, a human/mouse chimeric monoclonal antibody against the epidermal growth factor receptor (EGFR), is used as a single agent and in combination with chemotherapy or radiation therapy in metastatic colorectal cancer and locally advanced or metastatic head and neck squamous cell cancer. In cetuximab Summary of Product Characteristics (SPC), aseptic meningitis is mentioned as a rare nervous system disorder but with an unknown frequency. Rare but serious cancer drug-associated adverse reactions can be identified in the postmarketing experience after large numbers of patients have been exposed to the drug. As a rare complication, we report a case of aseptic meningitis associated with the first intravenous (I.V.) administration of cetuximab.

\section{Case presentation}

A 66-year-old woman, with a WHO performance status of 0 , history of chronic smoking, high blood pressure and atrial fibrillation, was diagnosed with a stage IVa locally advanced laryngeal squamous-cell carcinoma (cT3N2M0). She had neither history of headache nor previous allergic drug reactions. She received neoadjuvant chemotherapy by docetaxel, cisplatin and fluorouracil, with a marked tumor regression following three courses. She was then offered definitive external beam radiotherapy with concurrent weekly cetuximab. On her first cycle, she received routine premedication with dexchlorpheniramine $5 \mathrm{mg}$ I.V. followed by a loading dose of $400 \mathrm{mg} / \mathrm{m}^{2}$ cetuximab I.V. over $2 \mathrm{~h}(5 \mathrm{mg} / \mathrm{min})$ without developing any infusion reaction. Her usual medicines were rilmenidine, pantoprazole, fenofibrate, and acetaminophen. However, $4 \mathrm{~h}$ after completing cetuximab infusion, she was admitted to hospital with sudden headaches, photophobia, neck stiffness and vomiting without fever.

Cerebrospinal fluid (CSF) analysis showed a cloudy liquid with elevated protein $(1.5 \mathrm{~g} / \mathrm{L}$; normal range: $0.2-0.4 \mathrm{~g} / \mathrm{L}$ ), a red blood cell count of $6 / \mu \mathrm{L}$, and a leukocyte count of $4100 / \mu \mathrm{L}$ (normal range: $0-4 / \mu \mathrm{L}$ ),

\footnotetext{
* Correspondence: c.maritaz@gmail.com

${ }^{1}$ Department of Pharmacy, Saint-Joseph Hospital, Paris, France

Full list of author information is available at the end of the article
} 
$90 \%$ of them were neutrophils, $9 \%$ were lymphocytes, and $1 \%$ were monocytes. The glucose level in CSF was $3.16 \mathrm{mM}$ (normal range: $2.7-4.2 \mathrm{mM}$ ) with a glucose level in blood of $7.3 \mathrm{mM}$ (ratio 0.43 ). The white blood cell count was $7900 / \mu \mathrm{L}$ with $7000 / \mu \mathrm{L}$ neutrophils, and a C-reactive protein at $5.9 \mathrm{mg} / \mathrm{L}$ (normal range $<6.0 \mathrm{mg} / \mathrm{L}$ ). The patient was treated with empiric antibiotic therapy (ceftriaxone I.V.) for 7 days without corticosteroids and recovered neurologically within 8 days. Bacterial cultures remained negative. Viral analysis including a viral encephalitis panel was performed by polymerase chain reaction and remained negative. Repeat CSF analysis was initially planned 8 days after admission to the hospital but the lumbar puncture failed and was not repeated as the patient was well.

Symptoms resolution was reported by day 2. Radiation therapy was started 3 weeks after for 8 weeks and cetuximab was reintroduced 28 days after with a lower dose of $250 \mathrm{mg} / \mathrm{m}^{2}$. Methylprednisolone $80 \mathrm{mg}$ I.V. was added to dexchlorpheniramine $5 \mathrm{mg}$ I.V. and the infusion flow rate of cetuximab was decreased to $2 \mathrm{mg} / \mathrm{min}$. She tolerated it well and no side effects were reported all along the other additional infusions up to 10 weeks. At a follow-up of 18 months the patient is well with no evidence of tumor recurrence.

\section{Discussion}

The temporal association, clinical and laboratory findings strongly support the diagnosis of cetuximabinduced aseptic meningitis. As for our patient, most patients with aseptic meningitis are treated with antibiotics, pending identification of infectious agent and recover within 2 weeks, without any long-term neurological sequelae.

Distinction on clinical grounds alone is not possible, and the CSF pattern with neutrophilic pleocytosis may cause confusion with infectious meningitis. Resolution occurs several days after drug discontinuation. Diagnosis of aseptic meningitis is based on viral and bacterial CSF profiles remaining sterile.

Nonsteroidal anti-inflammatory drugs, antibiotics, intravenous immunoglobulins, antiepileptic drugs, and monoclonal antibodies (mainly tumor necrosis factor inhibitors) are the most frequent cause of druginduced meningitis. History of drug intake is crucial because there are no specific characteristics associated with a specific drug [1].

In order to try to understand the pathophysiology of aseptic meningitis due to cetuximab, we can draw similarities with aseptic meningitis occurring with I.V. immunoglobulin (IVIG) infusion [2-6]. The factors, which may predispose to the development of the meningitis, include fast infusion rates and a history of headaches. The symptoms of aseptic meningitis generally occur within $24 \mathrm{~h}$ of starting treatment. Theories of aseptic meningitis with IVIG have included an allergic hypersensitivity reaction or serum immunoglobulin crossing the blood brain barrier. Hence, this entry of serum immunoglobulin into the cerebrospinal fluid would be responsible for the inflammatory reaction. It has also been suggested that releasing histamine, serotonin, and prostaglandins could affect the meningeal microvasculature, such as in migraine mechanism [7].

The first occurrence of drug-induced aseptic meningitis related to cetuximab was reported in 2000 by Baselga et al. in a phase I clinical trial [8]. Since then, 7 other cases of cetuximab-induced aseptic meningitis have been described. Cetuximab was reintroduced successfully for three of them with an appropriate premedication and a slower infusion rate, one patient had a positive rechallenge without corticosteroid premedication $[9,10]$. Characteristics of the reported patients from the literature were compiled recently [11] and are now completed with a new one [12] and our present report in Table 1. Note that these adverse reactions always occurred during the first administration which may suggest a dose-related response, even though an idiosyncratic response in patients with risk factors is also possible. Surprisingly, there are no cases described in colorectal cancer whereas cetuximab is commonly being dosed at $500 \mathrm{mg} / \mathrm{m}^{2}$ (higher dose) every 2 weeks for a larger number of patients.

\section{Conclusions}

Cetuximab-induced aseptic meningitis should be known as a potential severe adverse drug reaction with corticosteroids introduced before loading dose and slowed infusion. This has been taken into account in the 2014 SPC renewal, as "Prior to the first infusion, patients must receive premedication with an antihistamine and a corticosteroid at least $1 \mathrm{~h}$ prior to administration of cetuximab. This premedication is recommended prior to all subsequent infusions " and "The initial dose should be given slowly and speed of infusion must not exceed $5 \mathrm{mg} /$ $\min (1 \mathrm{ml} / \mathrm{min})$. For the subsequent doses, the infusion rate must not exceed $10 \mathrm{mg} / \mathrm{min}(2 \mathrm{ml} / \mathrm{min}) »$. This report added to the others may serve as a reference for health practitioners managing cetuximab. Rechallenge with cetuximab after complete neurological resolution is feasible and should be attempted, especially when cetuximab is given in a curative intent. 
Table 1 Characteristics of the described cases of cetuximab-induced aseptic meningitis

\begin{tabular}{|c|c|c|c|c|c|c|c|c|}
\hline Case, Date & Age range & $\begin{array}{l}\text { Indication for } \\
\text { cetuximab }\end{array}$ & $\begin{array}{l}\text { Cetuximab dose } \\
\text { (duration), } \\
\text { premedication }\end{array}$ & $\begin{array}{l}\text { Symptoms (time onset), } \\
\text { imaging }\end{array}$ & Initial CSF analysis & $\begin{array}{l}\text { Follow-up CSF } \\
\text { analysis }\end{array}$ & Treatment, recovery & Rechallenge \\
\hline $1,2000[8]$ & $N / R$ & $N / R$ & $100 \mathrm{mg} / \mathrm{m}^{2}$ & $N / R$ & $\mathrm{~N} / \mathrm{A}$ & N/A & $N / R$ & $N / R$ \\
\hline $2,2009[10]$ & $40-49$ & $\begin{array}{l}\text { Recurrent laryngeal } \\
\text { squamous cell } \\
\text { carcinoma }\end{array}$ & $\begin{array}{l}400 \mathrm{mg} / \mathrm{m}^{2} \text { (first } \\
\text { administration } 2 \mathrm{~h} \text { ), } \\
\text { diphenhydramine } \\
50 \mathrm{mg} \mathrm{IV}\end{array}$ & $\begin{array}{l}\text { Frontal headache, } 38.9^{\circ} \mathrm{C} \\
\text { fever (few hours after } \\
\text { infusion), N/R }\end{array}$ & $\begin{array}{l}2300 / \mu l \text { with } 98 \% \\
\text { neutrophils, protein } \\
1.04 \mathrm{~g} / \mathrm{L} \text {, normal } \\
\text { glucose level, } \\
\text { negative cultures }\end{array}$ & $\begin{array}{l}\text { "Resolution of } \\
\text { neutrophilic } \\
\text { pleocytosis", } \\
\text { normal protein } \\
\text { levels (day 4) }\end{array}$ & $\begin{array}{l}\text { Empirical antibiotic } \\
\text { treatment, acyclovir, } \\
\text { recovery N/R }\end{array}$ & $\begin{array}{l}\text { Negative rechallenge after } 1 \\
\text { week ( } 250 \mathrm{mg} / \mathrm{m}^{2} \text {, premedication: } \\
\text { dexamethasone, diphenhydramine) } \\
\text { without adverse events }\end{array}$ \\
\hline $3,2009[10]$ & $40-49$ & $\begin{array}{l}\text { Locally advanced } \\
\text { squamous cell } \\
\text { carcinoma of right } \\
\text { tonsil }\end{array}$ & $\begin{array}{l}400 \mathrm{mg} / \mathrm{m}^{2} \text { (first } \\
\text { administration } 2 \mathrm{~h} \text { ), } \\
\text { diphenhydramine } \\
50 \mathrm{mg} \text { IV }\end{array}$ & $\begin{array}{l}\text { Severe frontal headache, } \\
39.4^{\circ} \mathrm{C} \text { fever, neck } \\
\text { stiffness, photophobia } \\
\text { (about } 8 \text { h after infusion), } \\
\text { N/R }\end{array}$ & $\begin{array}{l}2267 / \mu \mathrm{l} \text { with } 90 \% \\
\text { neutrophils, protein } \\
1.46 \mathrm{~g} / \mathrm{L}, \text { normal } \\
\text { glucose level, } \\
\text { negative cultures }\end{array}$ & $\begin{array}{l}\text { "No white blood } \\
\text { cells", elevated } \\
\text { but improved } \\
\text { protein }(0.69 \mathrm{~g} / \mathrm{L})\end{array}$ & $\begin{array}{l}\text { Empirical antibiotic } \\
\text { treatment, acyclovir, } \\
\text { dexamethasone, } \\
\text { recovery from } \\
\text { meningeal } \\
\text { symptoms after } \\
12 \text { days }\end{array}$ & $\begin{array}{l}\text { Negative rechallenge after } 2 \text { weeks } \\
\text { ( } 250 \mathrm{mg} / \mathrm{m}^{2} \text {, premedication: } \\
\text { dexamethasone, diphenhydramine, } \\
\text { famotidine) without adverse events }\end{array}$ \\
\hline $4,2010[13]$ & 70-79 & NSCLC (stage IIIA) & $\begin{array}{l}400 \mathrm{mg} / \mathrm{m}^{2} \text { (first } \\
\text { administration, } \\
\text { duration } \mathrm{N} / \mathrm{R} \text { ), N/R }\end{array}$ & $\begin{array}{l}\text { Severe headache, nausea, } \\
\text { vomiting, neck stiffness } \\
\text { (few hours after infusion), } \\
\text { brain CT scan normal }\end{array}$ & $\begin{array}{l}528 / \mu l \text { with } 87 \% \\
\text { neutrophils, " } \\
\text { modestly elevated } \\
\text { protein", normal } \\
\text { glucose level }\end{array}$ & N/A & $\begin{array}{l}\text { Empirical antibiotic } \\
\text { treatment (stopped } \\
\text { after infection was } \\
\text { ruled out), recovery } \\
\text { without neurological } \\
\text { sequelae }\end{array}$ & $N / R$ \\
\hline $5,2010[13]$ & $50-59$ & Metastatic NSCLC & $\begin{array}{l}400 \mathrm{mg} / \mathrm{m}^{2} \text { (first } \\
\text { administration, } \\
\text { duration } \mathrm{N} / \mathrm{R} \text { ), } \mathrm{N} / \mathrm{R}\end{array}$ & $\begin{array}{l}\text { Acute encephalopathy } \\
\text { (few hours after infusion), } \\
\text { brain CT scan and MRI } \\
\text { normal }\end{array}$ & $\begin{array}{l}\text { cell count and } \\
\text { fraction of } \\
\text { neutrophils N/A, } \\
\text { protein } 1.16 \mathrm{~g} / \mathrm{L} \text {, } \\
\text { glucose } 2.8 \\
\mathrm{mmol} / \mathrm{L} \text {, negative } \\
\text { cultures }\end{array}$ & N/A & $\begin{array}{l}\text { Empirical antibiotic } \\
\text { treatment (stopped } \\
\text { after infection was } \\
\text { ruled out), recovery } \\
\text { within several days }\end{array}$ & $N / R$ \\
\hline $6,2012[9]$ & $50-59$ & $\begin{array}{l}\text { Squamous maxillary } \\
\text { cancer (stage IVb) }\end{array}$ & $\begin{array}{l}400 \mathrm{mg} / \mathrm{m}^{2} \text { (first } \\
\text { administration), } \\
\text { diphenhydramine } \\
50 \mathrm{mg} \text { IV }\end{array}$ & $\begin{array}{l}\text { Frontal headache, neck } \\
\text { discomfort, } 39.9^{\circ} \mathrm{C} \text { fever } \\
\text { (few hours after infusion), } \\
\text { brain CT scan normal }\end{array}$ & $\begin{array}{l}1025 / \mu l \text { with } 92 \% \\
\text { neutrophils, protein } \\
1.65 \mathrm{~g} / \mathrm{L} \text {, normal } \\
\text { glucose level, } \\
\text { negative bacterial } \\
\text { culture, PCR (HSV) } \\
\text { negative }\end{array}$ & N/A & $\begin{array}{l}\text { Empirical antibiotic } \\
\text { treatment, resolution } \\
\text { of symptoms - no } \\
\text { complications. }\end{array}$ & $\begin{array}{l}\text { Positive rechallenge after } 4 \text { weeks } \\
\left(250 \mathrm{mg} / \mathrm{m}^{2}\right) \text {, recurrent CSF } \\
\text { pleiocytosis }(715 / \mu \text { l, } 93 \% \\
\text { neutrophils), protein } 1.22 \mathrm{~g} / \mathrm{L} \text {, } \\
\text { premedication: diphenhydramine. } \\
\text { Rechallenge three and following } \\
\text { without adverse events. }\end{array}$ \\
\hline 7, $2015[11]$ & $60-69$ & $\begin{array}{l}\text { Recurrent advanced } \\
\text { oropharyngeal } \\
\text { squamous cell } \\
\text { carcinoma }\end{array}$ & $\begin{array}{l}400 \mathrm{mg} / \mathrm{m}^{2} \text { (first } \\
\text { administration, } \\
2 \mathrm{~h} \text { ), clemastine } \\
2 \mathrm{mg} \text { oral }\end{array}$ & $\begin{array}{l}\text { Headache, mutism, } \\
\text { hypertension, neck } \\
\text { stiffness, } 39.2^{\circ} \mathrm{C} \text { fever } \\
\text { (about } 9 \mathrm{~h} \text { after infusion), } \\
\text { brain } \mathrm{CT} \text { scan and MRI } \\
\text { non-diagnostic }\end{array}$ & $\begin{array}{l}1413 / \mu l \text { with } 92 \% \\
\text { neutrophils, protein } \\
1.79 \mathrm{~g} / \mathrm{L} \text {, normal } \\
\text { glucose level } 3.5 \\
\text { mmol } / \mathrm{L} \text {, negative } \\
\text { cultures and } \\
\text { serologies }\end{array}$ & $\begin{array}{l}\text { Cell count } 1 / \mu \mathrm{l}, \\
\text { protein } 0.68 \mathrm{~g} / \mathrm{L}, \\
\text { normal glucose } \\
\text { level } 4.0 \mathrm{mmol} / \mathrm{L}\end{array}$ & $\begin{array}{l}\text { Empirical antibiotic } \\
\text { treatment, } \\
\text { dexamethasone } \\
\text { (stopped after } \\
\text { infection was ruled } \\
\text { out), myoclonic } \\
\text { jerks and NCSE } \\
\text { after } 3 \text { days, recovery } \\
\text { within } 14 \text { days }\end{array}$ & $\begin{array}{l}\text { The patient refused } \\
\text { rechallenge. }\end{array}$ \\
\hline
\end{tabular}


Table 1 Characteristics of the described cases of cetuximab-induced aseptic meningitis (Continued)

\begin{tabular}{|c|c|c|c|c|c|c|c|c|}
\hline $8,2015[12]$ & $50-59$ & $\begin{array}{l}\text { Tonsillar squamous } \\
\text { cell cancer }\end{array}$ & $\begin{array}{l}400 \mathrm{mg} / \mathrm{m}^{2} \text { (first } \\
\text { administration) }\end{array}$ & $\begin{array}{l}\text { Frontal headache (10/10 } \\
\text { in severity), fever ( } 1 \mathrm{~h} \\
\text { after infusion), brain CT } \\
\text { scan }\end{array}$ & $\begin{array}{l}473 / \mu l \text { with } 80 \% \\
\text { neutrophils in tube } \\
1 \text { and } 500 / \mu l \text { with } \\
62 \% \text { neutrophilsin } \\
\text { tube } 4 . \\
150 \text { and } 50 \text { cells } / \mu \mathrm{L} \\
\text { red blood cells, } \\
\text { protein } 1,28 \mathrm{~g} / \mathrm{L} \text {, } \\
\text { normal glucose level }\end{array}$ & $\mathrm{N} / \mathrm{A}$ & $\begin{array}{l}\text { Empirical antibiotic } \\
\text { treatment for } 4 \text { days } \\
\text { (stopped after } \\
\text { infection was ruled } \\
\text { out). Symptomatic } \\
\text { improvement after } \\
2 \text { days and recovery } \\
\text { within } 4 \text { days }\end{array}$ & $\begin{array}{l}\text { Negative rechallenge after } 7 \text { days, } \\
\text { the patient received a second } \\
\text { dose of cetuximab at } 250 \mathrm{mg} / \mathrm{m} 2 \\
\text { without adverse events. }\end{array}$ \\
\hline $\begin{array}{l}\text { 9, } 2015 \\
\text { Present case }\end{array}$ & $60-69$ & $\begin{array}{l}\text { Locally advanced } \\
\text { laryngeal squamous } \\
\text { cell carcinoma }\end{array}$ & $\begin{array}{l}400 \mathrm{mg} / \mathrm{m}^{2} \text { (first } \\
\text { administration, } 2 \mathrm{~h} \text { ), } \\
\text { dexchlorpheniramine } \\
5 \mathrm{mg} \mathrm{IV}\end{array}$ & $\begin{array}{l}\text { Headache, photophobia, } \\
\text { neck stiffness, vomiting, } \\
\text { nausea (few hours after } \\
\text { infusion), N/A }\end{array}$ & $\begin{array}{l}\text { Leukocytes count } \\
4100 / \mu \mathrm{L} \text { with } 90 \% \\
\text { of neutrophils, } 6 / \mu \mathrm{L} \\
\text { red blood cells, } \\
\text { protein } 1.5 \mathrm{~g} / \mathrm{L} \text {, } \\
\text { normal glucose } \\
\text { level in } 3.16 \mathrm{mmol} / \mathrm{L} ., \\
\text { negative viral and } \\
\text { bacterial cultures. }\end{array}$ & N/A & $\begin{array}{l}\text { Empirical antibiotic } \\
\text { treatment for } 7 \text { days, } \\
\text { recovery without } \\
\text { sequelae within } \\
\text { several days }\end{array}$ & $\begin{array}{l}\text { Negative rechallenge after } 28 \\
\text { days }\left(250 \mathrm{mg} / \mathrm{m}^{2}, \text { premedication: }\right. \\
\text { methylprednisolone, } \\
\text { dexchlorpheniramine) } \\
\text { without adverse events. }\end{array}$ \\
\hline
\end{tabular}




\section{Abbreviations}

EGFR, epidermal growth factor receptor; SPC, summary of product characteristics; IV, intravenous; WHO, World Health Organization; CSF, cerebrospinal fluid

\section{Acknowledgement}

We thank the nursing staff of the Department of Oncology at the SaintJoseph hospital in Paris for their skill in helping the patient presented in this case report.

\section{Funding}

This study was not funded by any outside source.

\section{Availability of data and materials}

The datasets supporting the conclusions of this article are included within the article.

\section{Authors' contributions}

$\mathrm{CMa}$ and $\mathrm{CM}$ e performed literature review and wrote the manuscript. $\mathrm{NBH}$ MJS, and GD contributed to the development of the report by revising it critically. All authors were involved in the management of the patient. All authors read and approved the final manuscript.

\section{Competing interests}

The authors declare that they have no competing interests.

\section{Consent for publication}

Written informed consent was obtained from the patient for publication of this Case report. A copy of the written consent is available for review by the Editor of this journal.

\section{Author details}

${ }^{1}$ Department of Pharmacy, Saint-Joseph Hospital, Paris, France. ${ }^{2}$ Department of Oncology, Saint-Joseph Hospital, Paris, France. ${ }^{3}$ Department of Oncology, University hospital of Lausanne, Lausanne, Switzerland.

Received: 22 September 2015 Accepted: 20 June 2016

Published online: 04 July 2016

\section{References}

1. Moris G, Giarca-Mono JC. The challenge of drug-induce meningitis revisited. JAMA Intern Med. 2014:174:1511-2.

2. Sekul EA, Cupler EJ, Dalakas MC. Aseptic meningitis associated with highdose intravenous immunoglobulin therapy: frequency and risk factors. Ann Intern Med. 1994;121:259-62.

3. Hamrock DJ. Adverse events associated with intravenous immunoglobulin therapy. Int Immunopharmacol. 2006;6:535-42.

4. Stiehm ER. Adverse effects of human immunoglobulin therapy. Transfus Med Rev. 2013;27:171-8

5. Mullane D, Williams L, Merwick A, et al. Drug induced aseptic meningitis caused by intravenous immunoglobulin therapy. Ir Med J. 2012;105:182-3.

6. Cherin P, Marie I, Michallet M, Pelus E, Dantal J, Crave JC, et al. Management of adverse events in the treatment of patients with immunoglobulin therapy: a review of evidence. Autoimmun Rev. 2016;15:71-81.

7. Wiles CM, Brown P, Chapel H, Guerrini R, Hughes RA, Martin TD, et al. Intravenous immunoglobulin in neurological disease: a specialist review. I Neurol Neurosurg Psychiatry. 2002;72:440-8.

8. Baselga J, Pfister D, Cooper MR, Cohen R, Burtness B, Boss M, et al. Phase I studies of anti-epidermal growth factor receptor chimeric antibody C225 alone and in combination with cisplatin. J Clin Oncol. 2000;18:904-14.

9. Emani MK, Zaiden Jr RA. Aseptic meningitis: a rare side effect of cetuximab therapy. J Oncol Pharm Pract. 2013;19:178-80.

10. Feinstein TM, Gibson MK, Argiris A. Cetuximab-induced aseptic meningitis. Ann Oncol. 2009:20:1609-10.

11. Ulrich A, Weiler S, Weller M, Rordorf T, Tarnutzer AA. Cetuximab induced aseptic meningitis. J Clin Neurosci. 2015:22:1061-3.

12. Prasanna D, Elrafei T, Shum E, Strakhan M. More than a headache: a case of cetuximab-induced aseptic meningitis. BMJ Case Rep. 2015;12:2015.

13. Nagovskiy N, Agarwal M, Allerton J. Cetuximab-induced aseptic meningitis. J Thorac Oncol. 2010:5:751.

\section{Submit your next manuscript to BioMed Central and we will help you at every step:}

- We accept pre-submission inquiries

- Our selector tool helps you to find the most relevant journal

- We provide round the clock customer support

- Convenient online submission

- Thorough peer review

- Inclusion in PubMed and all major indexing services

- Maximum visibility for your research

Submit your manuscript at www.biomedcentral.com/submit 